\title{
Children with chronic pain: Impact of sex and age on long-term outcomes
}

\author{
Andrea L. Martin ${ }^{a, b, *}$, Patricia A. McGrath ${ }^{a, b, c}$, Stephen C. Brown ${ }^{a, c}$, Joel Katz ${ }^{\text {b,c }}$ \\ a Anesthesia, Divisional Center for Pain Management and Research, The Hospital for Sick Children, Toronto, Ont., Canada \\ ${ }^{\mathrm{b}}$ Department of Psychology, York University, Toronto, Ont., Canada \\ ${ }^{c}$ Department of Anesthesia, The University of Toronto, Toronto, Ont., Canada
}

\begin{abstract}
The present study examined the long-term pain and disability outcomes of a pediatric chronic pain clinic cohort and evaluated whether these outcomes differed by age and sex. Patients were interviewed a mean of 3 years after their last appointment at a pediatric pain clinic. The cohort comprised 95 females and 48 males, aged 5-23 years when interviewed. Of the cohort, 62.2\% (67 females, 22 males) reported continuing pain. Females were significantly more likely than males to report continuing pain $(O R=2.9,95 \% C I=1.4-5.8, p=.005)$, use of health care $(O R=5.1,95 \% C I=1.4-18.5, p=.01)$, medication $(O R=4.7,95 \%$ $C I=1.3-16.9, p=.02)$ and non-drug methods of pain control $(O R=3.4,95 \% C I=1.3-9.2, p=.02)$. For patients whose pain had associated psychosocial factors, females $(76.4 \%)$ were significantly more likely than males $(21.4 \%)$ to report continuing pain $(O R=13.8,95 \% C I=3.3-58.4, p=.005)$. Finally, among patients still experiencing pain, the frequency of pain episodes increased significantly with age $(O R=1.3,95 \% C I=1.0-1.5, p=.02)$. Results indicate that chronic pain persists for many children despite treatment at specialized clinics. Females may be at higher risk for continuing pain and report greater use of health care, medication, and non-drug methods of pain control.
\end{abstract}

Keywords: Chronic pain; Children; Long-term outcomes; Sex differences; Age differences; Psychosocial factors

\section{Introduction}

Like adults, chronic pain in children is a complex phenomenon that may develop spontaneously or after disease, infection, injury, surgery, or from idiopathic causes. However, in contrast to the more extensive knowledge on the epidemiology of chronic pain in adults (Crombie et al., 1999), we lack epidemiological data on pain in children. In particular, few longitudinal, casecontrol and cohort studies have been conducted, so that we know very little about the natural history, risk factors, and impact of pain on the lives of children and

\footnotetext{
${ }^{*}$ Corresponding author. Tel.: $+14168137654 \times 2375$; fax: +1416 8132186.

E-mail address: amartin@yorku.ca (A.L. Martin).
}

their families (McGrath, 1999; Van Den Kerkhof and van Dijk, 2006). Recent population-based studies of children indicate that chronic pain is a continuing problem for some children, with musculoskeletal pain persisting for $63.5 \%$ of children at a 4 -year follow-up (ElMetwally et al., 2004), chronic benign pain persisting for $30 \%$ at a 2-year follow-up (Perquin et al., 2003), and widespread pain persisting for $29 \%$ at a 1 -year follow-up (Mikkelsson et al., 1999).

Similarly, clinical studies indicate that pain persists for certain children. Guidetti and Galli (1998) reported that headache in 100 children, 4-18 years of age, tended to remit $(34 \%)$ or improve $(45 \%)$ at an 8 -year clinical follow-up. In a 10-year clinical follow-up, Dooley and Bagnell (1995) reported $73 \%$ of children had continuing headache, although $82 \%$ were much improved. Further- 
more, in a clinical sample of 16 children, 6-12 years of age, with recurrent abdominal pain, 50\% reported continuing pain at a 10-year follow-up (Magni et al., 1987).

Many studies of chronic pain describe child, family, and environmental characteristics in an effort to identify the risk factors that may predispose children to develop pain. Female sex and increased age are important risk factors affecting lifetime prevalence rates (Balague et al., 1999; Perquin et al., 2000; Hakala et al., 2002) and long-term outcomes (El-Metwally et a1., 2004) of certain pains in population- and school-based cohorts of children. Several psychosocial factors affect pain intensity and pain-related disability, such as a child's emotions and a child and family's behaviors in response to pain (McGrath and Hillier, 2003). Accordingly, treatment emphasis for chronic pain has shifted from a disease specific focus to a more child-centered focus, with interdisciplinary pain teams addressing a child's pain from a broader psychosocial perspective (McGrath, 2005). Paradoxically, though, despite recognizing the multidimensional nature of pain, childhood chronic pain is almost exclusively classified according to physical diagnoses rather than considering the presence of psychosocial factors contributing to pain or disability.

In order to better understand chronic pain and to develop optimal interventions, longitudinal studies are needed to examine developmental changes in the transition from childhood to adolescence and adolescence to adulthood and to describe relevant biopsychosocial factors (McGrath and Goodman, 1991; McGrath, 1999; Perquin et al., 2000, 2003; Brattberg, 2004). The present descriptive cohort study assessed the long-term pain and disability outcomes for a clinic cohort of children with chronic pain and explored the impact of sex (the physiological male and female makeup), age and the presence of associated psychosocial factors on these outcomes.

\section{Methods}

\subsection{Study design}

In order to capture a diverse array of children with chronic pain, we surveyed the population of children who had been treated for chronic pain by a multidisciplinary team at a specialized pain clinic for children (Hospital for Sick Children, Toronto, Canada). When the study cohort was originally seen in clinic, our core team included a medical director (anesthesiologist), nurse clinician, physiotherapist, and psychiatrist. All children were assessed by the team, who then met privately to discuss the probable etiology and any psychosocial factors contributing to pain and disability. The multidisciplinary team diagnosed children's pain at the time of initial assessment in the pain clinic. The team developed a child-centered treatment regimen - usually comprised of medication (analgesics or adjuvant analgesics) and physiotherapy, with some children referred for relaxation training and some for cognitive behavioral therapy.
Post-discharge, at the time of data collection for the present study, children were further classified as to whether psychosocial factors had been associated with their pain and disability. This classification was based on a chart review of the team's findings of the initial pain interview-and subsequent treatment sessions. Examples of associated psychosocial factors included evidence of school or social withdrawal, pain/avoidance behaviors reinforced by family members, and that children exhibited anxiety or depression warranting referral to Psychiatry or Psychology.

Our objective in this study was to identify and interview a heterogeneous cohort of children with chronic pain, who had been assessed and managed according to "best practice", rather than to evaluate the efficacy of specific treatments for different pain conditions. The research protocol for the present study was approved by the Research Ethics Boards at The Hospital for Sick Children and York University.

\subsection{Subjects}

The eligible clinic cohort included 209 children and adolescents who had been assessed in the chronic pain clinic at The Hospital for Sick Children 1-6 years previously $(M=3.1$, $S D=1.3$ years). Current contact information was not available for 23 of the 209 patients. We contacted 186 patients by telephone; 148 agreed to participate (79.6\%), 29 did not return our calls, 3 had died (we do not know the cause of deaths), and 6 did not agree to participate in the follow-up survey.

\subsection{Pain outcomes interview}

Three trained assistants conducted telephone interviews with adolescents (previous patients) or parents for the patients who were younger than 16 years of age. The brief interview (10-15 min) comprised 10 questions, including both open-ended and supplied response questions.

Respondents (adolescent or parent) were first asked "When we saw you (your child) in the clinic you (he/she) had persistent type of pain." "Do you (Does he/she) still have this pain?" If respondents answered yes, they were asked to rate the frequency (supplied response: less than once a month, once a month, 1-2 times per week, several times (3-6 times) per week, daily, constantly) and intensity (0-10 numeric rating scale) of the current pain. Interviewers asked respondents whether the pain interfered with school, work, sports/recreation, and interactions with friends and family. They then asked whether the patient was currently using any non-drug methods of pain control or taking any pain medication. If respondents reported use of medication, interviewers asked about the type and frequency of use. Respondents were also asked if the patient had developed any new pain problems since they had been seen in the clinic and if they were currently seeing a health professional specifically for pain.

\subsection{Data analyses}

We calculated the proportions of children who continued to experience pain and those who no longer had pain for the cohort as a whole and by age, sex, and presence/absence of associated psychosocial factors. Means and standard deviations were calculated as descriptive measures of children's rat- 
ings of pain intensity and frequency distributions were generated for all categorical variables. Multiple logistic regression analyses were computed to explore age, sex, and an age $\times$ sex interaction as predictors for each outcome factor. Odds ratios $(\mathrm{OR})$ and $95 \%$ confidence intervals $(\mathrm{CI})$ are reported. Variables that did not contribute to the model were dropped to arrive at the final regression model. We also explored whether the presence or absence of associated psychosocial factors at the initial assessment predicted ongoing pain at follow-up. Multiple linear regression analysis was used to explore age- and sex-related differences in reported pain intensity as a continuous variable.

\section{Results}

Of the 148 respondents who agreed to participate, five patients were excluded from the data analyses: four due to the presence of multiple pain locations and one due to missing data. The final study cohort included 143 children and adolescents $(68.4 \%$ of the clinic cohort), 95 females and 48 males, aged 5-23 years $(M=16.7, S D=3.5$ years $)$ at the time of the follow-up interview. Of the 143 interviews, 86 $(60.1 \%)$ were conducted with adolescents (previous patients) and $57(39.9 \%)$ with parents. Pain diagnoses are listed in Table 1.

Chi-squared analysis did not reveal a significant difference in the proportion of males and females across the four largest diagnostic categories (abdominal pain, headache, musculoskeletal, other; $\chi^{2}(3)=2.2, p=.5$ ). An independent samples $t$-test did not show a significant difference in the mean age of males $(M=16.5, S D=3.1$ years $)$ and females $(M=16.9, S D=3.7$ years; $t(1,141)=0.6, p=.6)$. However, the mean number of follow-up years for males $(M=3.4, S D=1.4$ years $)$

Table 1

Primary diagnosis after initial assessment in chronic pain clinic

\begin{tabular}{lrr}
\hline Diagnosis & \multicolumn{2}{c}{$\%$} \\
\hline Abdominal pain - identified disease & 11 & 7.7 \\
Abdominal pain - post-surgical & 4 & 2.8 \\
Abdominal pain - recurrent & 10 & 6.9 \\
Chronic pain and disability & 8 & 5.6 \\
CRPS type 1 & 13 & 9.1 \\
Fibromyalgia & 2 & 1.4 \\
Headache - chronic daily & 8 & 5.6 \\
Headache - mixed & 2 & 1.4 \\
Headache - identified disease & 3 & 2.1 \\
Headache - post-traumatic & 1 & 0.7 \\
Musculoskeletal - idiopathic & 29 & 20.3 \\
Musculoskeletal - identified disease & 11 & 7.7 \\
Musculoskeletal - post-infectious & 3 & 2.1 \\
Musculoskeletal - post-surgical & 14 & 9.8 \\
Musculoskeletal - post-traumatic & 14 & 9.8 \\
Neuromuscular - identified disease & 4 & 2.8 \\
Phantom limb pain & 6 & 4.2 \\
Total & 143 & 100.0 \\
\hline
\end{tabular}

${ }^{a}$ CRPS type 1 , complex regional pain syndrome type 1 . was significantly higher than that for females $(M=2.9, S D=1.3$ years; $t(1,141)=2.1, p=.04)$.

\subsection{Proportion of patients with continuing chronic pain}

More than half the cohort $(62.2 \%)$ reported continued pain at follow-up. A significantly greater proportion of females $(70.5 \%)$ than males $(45.8 \%)$ reported continued pain; females were nearly three times more likely than males to report chronic pain $(O R=2.9,95 \%$ $C I=1.4-5.8, p=.005$ ). Table 2 presents the number of patients with continuing pain by age, diagnosis and associated psychosocial factors. We were not able to evaluate the impact of specific diagnoses (Table 1) on pain outcomes given the many different diagnoses. However, chi-squared analysis did not reveal a significant difference in the proportion of patients with or without continuing pain across the four largest diagnostic categories (abdominal pain, headache, musculoskeletal, other; $\chi^{2}(3)=2.2, p=.5$ ).

\subsection{Pain characteristics}

\subsubsection{Pain frequency}

Pain frequency, recorded with a supplied response format, was coded into one of two categories based on a median split: low frequency (less than once a month, once a month, 1-2 times per week) or high frequency (several times (3-6 times) per week, daily, constantly). Of the 89 patients still experiencing pain, more than half $(60.2 \%)$ rated the frequency of their pain episodes in the high frequency category (Fig. 1). Six patients were unable to rate the frequency of their pain (five patients

Table 2

Pain outcomes by age, diagnosis and presence of associated psychosocial factors at initial assessment

\begin{tabular}{lcl}
\hline & Continuing pain & No pain \\
\hline Total, \% of all $(n)$ & $62.2 \%(89)$ & $37.8 \%(54)$ \\
Age, \% of age group $(n)$ & & \\
$0-11$ years & $42.9 \%(6)$ & $57.1 \%(8)$ \\
$12-14$ years & $64.3 \%(18)$ & $35.7 \%(10)$ \\
$15-17$ years & $58.7 \%(27)$ & $41.3 \%(19)$ \\
$18-20$ years & $63.4 \%(26)$ & $36.6 \%(15)$ \\
$21-23$ years & $85.7 \%(12)$ & $14.3 \%(2)$ \\
Diagnosis, \% of diagnostic category $(n)$ & \\
Abdominal pain & $52.0 \%(13)$ & $48.0 \%(12)$ \\
Musculoskeletal pain & $67.6 \%(48)$ & $32.4 \%(23)$ \\
Headache & $57.1 \%(8)$ & $42.9 \%(6)$ \\
Other & $60.6 \%(20)$ & $39.4 \%(13)$
\end{tabular}

Presence of associated psychosocial factors at initial assessment, $\%(n)^{*}$ Males $\quad 21.4 \%(3) \quad 78.6 \%(11)$ \begin{tabular}{ll} 
Females & $76.4 \%(42)$ \\
\hline
\end{tabular}

${ }^{*} p=.005$. Of children with associated psychosocial factors at initial assessment, significantly more females than males reported pain at follow-up. 


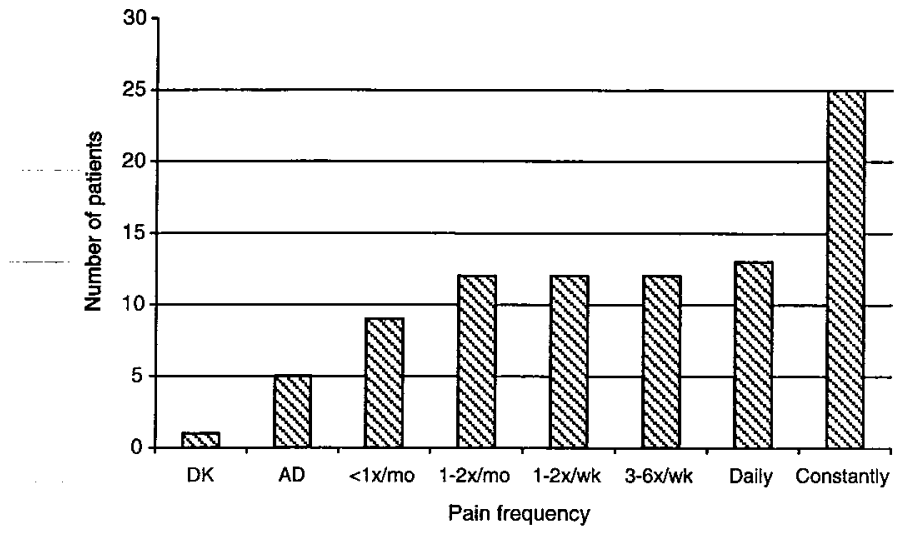

Fig. 1. Reported pain frequency for patients currently experiencing chronic pain. DK, do not know; AD, activity dependent.

felt their pain was entirely activity dependent and one patient reported that he did not know how often he experienced pain). Pain frequency did not differ by sex. However, for those still experiencing pain, the pain frequency increased with age after controlling for pain intensity; patients were 1.3 times more likely to report pain in the high frequency category $(3-6 \times /$ wk - constantly) for every 1 year increase in age $(O R=1.3$, $95 \% C I=1.0-1.5, p=.02$ ). For example, 20-year-olds were 5.2 times more likely to report pain in the high frequency category than were 16-year-olds.

\subsubsection{Pain intensity}

The mean pain intensity score (0-10 numeric rating scale) for average pain was $6.1(S D=2.1)$. Mean average pain intensity ratings did not differ significantly by age or by sex.

\subsection{Impact of chronic pain}

Of the 89 patients still experiencing pain, $67.4 \%$ reported the pain interfered with their life in at least one of the following categories: sports and recreation, ability to attend school, ability to go out with friends, ability to participate in family activities, and for those patients working in a job, ability to go to work (Fig. 2). There were no differences in pain-related interference by sex or age.

\subsection{Current pain interventions}

\subsubsection{Non-drug pain control methods}

More than half $(62.9 \%)$ of those still experiencing pain reported current use of non-drug pain control methods such as massage therapy, relaxation techniques, physiotherapy, acupuncture and hypnosis (Fig. 3). Significantly more females (70.2\%) than males $(40.9 \%)$ reported use of non-drug methods of pain control; females were three times more likely than males to

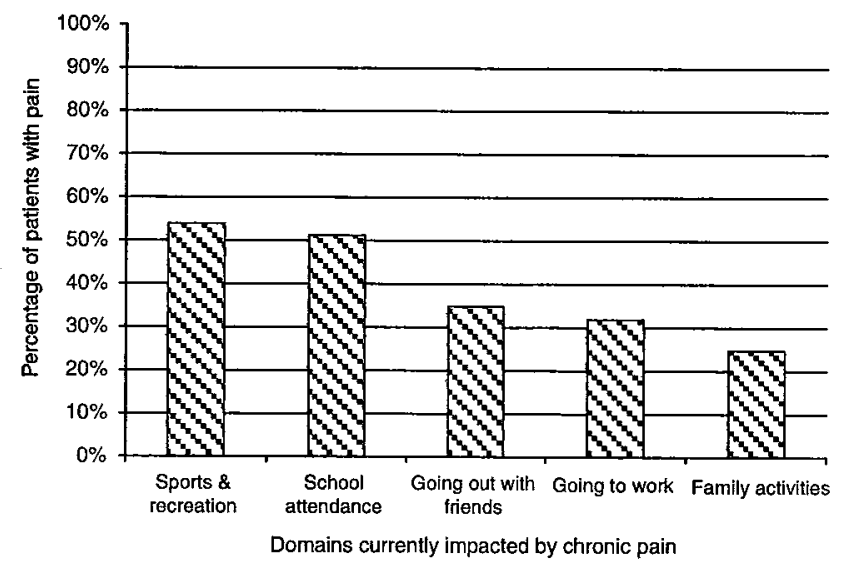

Fig. 2. Areas of pain-related interference for patients currently experiencing chronic pain.

use non-drug methods $(O R=3.4,95 \% C I=1.3-9.2$, $p=.02$ ). Use of non-drug pain control methods did not differ by age.

\subsubsection{Pain medication}

The majority $(70.8 \%)$ of those still experiencing pain reported taking pain medication (Fig. 3). Of those using medication, $38.1 \%$ reported taking medication "every time it hurts" and $61.9 \%$ reported "sometimes"/"hardly ever" taking medication for their pain. Logistic regression analyses revealed that after controlling for pain intensity, significantly more females $(76.1 \%)$ than males $(54.5 \%)$ reported taking pain medication; females were nearly five times more likely than males to take medication $(O R=4.7,95 \% C I=1.3-16.9, p=.02)$. Use of pain medication did not differ by age.

\subsection{New pain problems}

Since being discharged from the clinic, $39.3 \%$ of those still experiencing their original chronic pain ( 35 patients)

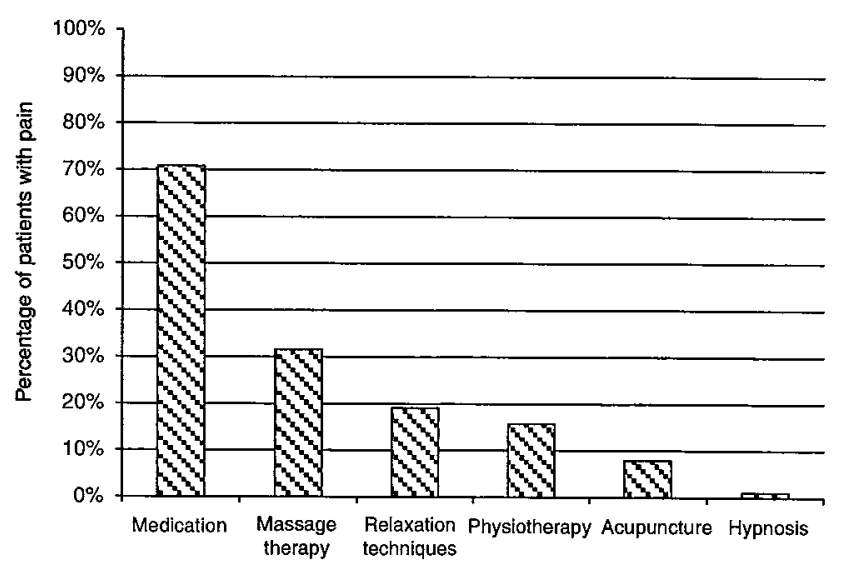

Fig. 3. Percentage of patients reporting current use of medication and non-drug pain control methods. 
and $18.5 \%$ of those who no longer had their original chronic pain (10 patients) had developed a new chronic pain. There were no differences by sex or age in the development of new pain problems.

\subsection{Currently receiving treatment specifically for pain}

One-third of children who were experiencing pain at follow-up, either a continuation of their old pain or the development of a new pain, were currently seeing a health care professional specifically for pain; a significantly greater proportion of these were female $(38.9 \%)$ than male $(11.1 \%)$. Females were five times more likely than males to be receiving treatment for their pain $(O R=5.1,95 \% C I=1.4-18.5, p=.01)$. Age did not impact whether or not a patient was currently receiving treatment for their pain.

\subsection{Associated psychosocial factors and ongoing pain}

Table 2 shows the number and percent of male and female patients with psychosocial factors present that reported ongoing pain. Logistic regression analysis was conducted with sex, age and presence of associated psychosocial factors as the predictors and continued pain at follow-up as the outcome. There was a significant interaction between sex and presence of associated psychosocial factors such that among those children whose initial pain had been associated with psychosocial factors, females $(76.4 \%)$ were nearly 14 times more likely than males $(21.4 \%)$ to continue to suffer pain $(O R=13.8$, $95 \% C I=3.3-58.4, p=0.005$ ).

\section{Discussion}

In this study, we interviewed a clinic cohort of children who had been diagnosed with chronic pain a mean of 3 years after their last appointment at a specialized pain clinic for children. Our high response rate of $79.6 \%$ is within the normative range $(M=60 \%$, $S D=21$ ) for surveys published in medical journals (Asch et al., 1997). The present study captured 148 patients $(70.8 \%)$ from the original clinic cohort $(N=209)$. We were unable to locate current contact information for $11 \%$ of our patients; however, due to a complete lack of contact with this group, we do not have any reason to believe that they were systematically different from the group of responders. As such, the pain and disability outcomes found are considered representative of the cohort under study.

Results indicate that a high proportion of children diagnosed with chronic pain continue to experience pain for a prolonged period. Sixty-two percent of the sample reported pain at follow-up, 1-6 years after discharge from the chronic pain clinic. These findings are consistent with those of El-Metwally et al. (2004) who found that $64 \%$ of children with musculoskeletal pain reported having pain at least once a week at a 4-year populationbased follow-up.

In the present study, over half of those with continuing pain reported pain that was highly frequent and adversely impacted their daily activities. Despite the presence of pain in over half the sample, use of health care resources was modest; only 31 of the 99 individuals with pain were receiving treatment. A similar overall decline in health care utilization was found in Perquin et al.'s, 2003 2-year population-based follow-up of 254 children (0-18 years of age) with chronic benign pain.

There were five main sex differences in pain outcomes for children in the present study. Females were significantly more likely than males to report continuing pain, to use medication and non-drug pain control methods, and to receive treatment specifically for their pain. Furthermore, across the subset of children whose pain was appreciably associated with psychosocial factors, females were significantly more likely than males to report continuing pain.

Several factors, alone or in combination, may account for these observed sex differences. One possibility is that these findings indicate a heightened vulnerability in females. Perquin et al. (2003) found that females were two times more likely than males to report chronic benign pain at follow-up. Many other population-based follow-up studies of children have also reported higher rates of chronic pain in females than in males (Balague et al., 1999; Perquin et al., 2000; Hakala et al., 2002; Brattberg, 2004). Additionally, the greater use of medication and/or non-drug methods among females, despite comparable pain intensity scores to males, suggests that females may have experienced more intense or frequent pain than males and used pain control methods to reduce pain to comparable levels.

The present findings also may reflect a gender difference in North American society in the report of pain: females may be more willing to report pain and to engage in non-drug pain control strategies and/or may find these strategies more effective than males. Males are often expected to be strong and brave. Consequently, they may endure a greater amount of pain before taking medication. In contrast, females are often expected to be weaker and to express themselves and thus may exhibit more help-seeking behavior (Zeman and Garber, 1996) and receive more treatment for their pain.

Furthermore, the higher proportion of females using non-drug pain control methods may be due to a differential pattern in parents' use of these strategies with daughters versus sons. A study examining the use of non-pharmacological methods to alleviate postoperative pain in children found that parents were more likely to use imagery, relaxation, touch and positive reinforcement when caring for their female children compared with their male children (Polkki et al., 2002). 
However, it is also possible that the types of non-drug methods with which individuals were prompted in the present study (massage therapy, relaxation techniques, physiotherapy, acupuncture and hypnosis) are biased towards the kinds of strategies females use. Thus, there may be other non-drug methods that boys use that were not given as examples (e.g., playing videogames or sports). This requires further study.

There were no significant sex differences in reported pain frequency and intensity, self-reported impact of pain on the child's life, the frequency with which children reported taking medication or the development of any new pain problems. With respect to the impact of age on long-term outcomes, logistic regression analyses revealed that for those still experiencing pain, the frequency of pain episodes increased with age after controlling for pain intensity. Age was not a significant predictor for any of the other pain outcomes. One might expect older age to be associated with continued pain or the development of new pain based on previous research reporting an increase in the prevalence of back pain (Burton et al., 1996; Balague et al., 1999), neck pain (Hakala et al., 2002), headache, abdominal pain and limb pain (Perquin et al., 2000) with age. However, this pattern was not the case for the cohort in the present study.

Interdisciplinary teams consistently assess the presence of contributing psychosocial factors to children's pain and disability (Berde and Solodiuk, 2003). However, studies have not evaluated whether these factors influence the long-term outcome of continuing pain. Our exploratory analyses indicate that females with psychosocial factors (i.e. evidence of school or social withdrawal, pain/avoidance behaviors reinforced by family members, and anxiety or depression warranting referral to Psychiatry or Psychology) contributing to their pain at the time of treatment are at much greater risk for continuing pain than males. Interestingly, our data also suggest that males with psychosocial factors are more likely to report no pain at follow-up. It is unclear what this sex or gender difference means. It is possible that males and females differed with respect to following recommendations for receiving psychological assistance or differed with respect to the family's interpretation of the impact of psychosocial factors (e.g., parents were more protective or solicitous of females than males). In contrast to females, males whose pain was judged to be associated with psychosocial factors may have been encouraged more to ignore the pain once families understood that they would not be harmed by resuming their activities or males may have developed other problems that we did not measure in the present study (e.g., school truancy, acting out, depression, anxiety). Long-term, prospective studies are required to better evaluate and understand the differential impact of psychosocial factors on males and females with chronic pain.
There are three limitations to the present study. The first is our use of parent interviews for the younger (515-year-old) patients as opposed to the self-report "gold standard" ratings used in the older (16-24-year-old) patients. The second limitation is our inability to evaluate outcomes by diagnostic categories due to the heterogeneous clinical cohort and the resulting small cell sizes for individual diagnoses. Spurious findings due to diagnostic differences are unlikely, since there was an even distribution of males and females across the four largest diagnostic categories (abdominal pain, headache, musculoskeletal and other), suggesting that the sex differences observed reflect true differences between males and females and not differences due to diagnoses. Finally, the determination that psychosocial factors were or were not appreciably associated with the patients' pain was based on an investigation of patient records at the time of follow-up and not at the time of assessment and treatment. The post hoc nature of this classification raises issues with respect to the accuracy and validity of the data. However, it is important to note this determination was made without the knowledge of the patients' status with respect to pain at follow-up.

In summary, 1-6 years after discharge from a chronic pain clinic, more than half the cohort reported continuing pain that was frequent, disabling, and necessitated active pain control interventions. The large proportion of children reporting pain at follow-up indicates the need for prospective research evaluating the effects of treatment (specific type and quantity) on long-term outcomes in clinic cohorts. When psychosocial factors are identified as contributing to children's pain and disability, teams should ensure that parents and children understand the role of these factors and the importance of psychological therapies, in addition to physical and pharmacological therapies (Lindley et al., 2005). Furthermore, given the modest use of health care resources, despite continuing pain in more than half the sample, it would be interesting to investigate the factors that predict return to clinic.

Our results indicate that females are significantly more likely than males to have chronic pain problems that continue into adolescence and adulthood. It is essential that pain management programs address children's pain from a biopsychosocial model, addressing the psychosocial factors that affect pain as well as the underlying physical etiology. We must also strive to identify the differential risk factors for chronic pain both developmentally and by sex and gender, so as to address those factors in treatment regimens.

\section{Acknowledgements}

This work was supported by New Emerging Team Grant GHL-63209 from the Institute of Gender and 
Health, Canadian Institutes of Health Research (CIHR) awarded to Dr. Patricia McGrath, Ontario, Canada. Joel Katz is supported by a Canada Research Chair in Health Psychology at York University. This paper is derived, in part, from Andrea Martin's Masters Thesis.

\section{References}

Asch DA, Jedrziewski $\mathrm{K}$, Christakis NA. Response rates to mail surveys published in medical journals. $\mathrm{J}$ Clin Epidemiol 1997;50:1129-36.

Balague F, Troussier B, Salminen JJ. Non-specific low back pain in children and adolescents: risk factors. Eur Spine J 1999;8:429-38.

Berde CB, Solodiuk J. Multidisciplinary programs for management of acute and chronic pain in children. In: Schechter NL, Berde CB, Yaster M, editors. Pain in infants, children \& adolescents. Baltimore, MD: Williams \& Wilkins; 2003. p. 471-86.

Brattberg G. Do pain problems in young school children persist into early adulthood? A 13-year follow-up. Eur J Pain 2004;8:187-99.

Burton AK, Clarke RD, McClune TD, Tillotson KM. The natural history of low back pain in adolescents. Spine 1996;21(20):2323-8.

Crombie IK, Croft PR, Linton SJ, LeResche L, VonKorff M, editors. Epidemiology of Pain. Seattle, WA: IASP Press; 1999.

Dooley $\mathbf{J}$, Bagnell A. The prognosis and treatment of headaches in children - a 10-year follow-up. Can J Neurol Sci 1995;22(1):47-9.

El-Metwally A, Salminen JJ, Auvinen A, Kautiainen H, Mikkelsson M. Prognosis of non-specific musculoskeletal pain in preadolescents: a prospective 4-year follow-up study till adolescence. Pain 2004;110:550-9.

Guidetti V, Galli F. Evolution of headache in childhood and adolescence: an 8-year follow-up. Cephalalgia 1998;18:449-54.

Hakala P, Rimpela A, Salminen JJ, Virtanen SM, Rimpela M. Back, neck, and shoulder pain in finnish adolescents: National cross sectional surveys. BMJ 2002;325(7367):743-6.
Lindley KJ, Glaser D, Milla PJ. Consumerism in healthcare can be detrimental to child health: lessons from children with functional abdominal pain. Arch Dis Child 2005;90:335-7.

Magni G, Pierri M, Donzelli F. Recurrent abdominal pain in children: a long-term follow-up. Eur J Pediatr 1987;146(1):72-4.

McGrath PA. Chronic pain in children. In: Crombie IK, Croft PR, Linton SJ, LeResche L, VonKorff M, editors. Epidemiology of pain. Seattle, WA: IASP Press; 1999. p. 81-101.

McGrath PA. Children - 'Not simply little adults'. In: Merskey H, Loeser JD, Dubner R, editors. The paths of pain: 19752005. Seattle, WA: IASP Press; 2005. p. 433-46.

McGrath PA, Hillier LM. Modifying the psychological factors that intensify children's pain and prolong disability. 2nd ed. In: Schechter NL, Berdes CB, Yaster M, editors. Pain in infants, children, and adolescents. Philadelphia, PA: Lippincott Williams \& Wilkins; 2003. p. 85-104.

McGrath PJ, Goodman JE. The epidemiology of pain in children and adolescents: a review. Pain 1991;46:247-64.

Mikkelsson M, Sourander A, Salminen JJ, Kautiainen H, Piha J. Widespread pain and neck pain in schoolchildren. A prospective one-year follow-up study. Acta Paediatr 1999;88:1119-24.

Perquin CW, Hazebroek-Kampschreur AAJM, Hunfeld JAM, Bohnen AM, van Suijlekom-Smit LWA, Passchier J, van der Wouden JC. Pain in children and adolescents: a common experience. Pain 2000;87:51-8.

Perquin CW, Hunfeld JAM, Hazebroek-Kampschreur AAJM, van Suijlekom-Smit LWA, Passchier J, Koes BW, van der Wouden JC. The natural course of chronic benign pain in childhood and adolescence; a two-year population-based follow-up study. Eur J Pain 2003; 7:551-9.

Polkki T, Vehvilainen-Julkunen K, Pietila AM. Parents' roles in using non-pharmacological methods in their child's postoperative pain alleviation. J Clin Nurs 2002;11:526-36.

Van Den Kerkhof EG, van Dijk A. Prevalence of chronic pain disorders in children. In: Schmidt RF, Willis WD, editors. Encyclopedia of Pain, Vol. 3. New York: Springer Verlag; 2006. p. $366-8$.

Zeman J, Garber J. Display rules for anger, sadness, and pain: it depends on who is watching. Child Dev 1996;67:957-73. 